\title{
Interactive comment on "Using wavelet transform to analyse on-road mobile measurements of air pollutants: a case study to evaluate vehicle emission control policies during the 2014 APEC summit” by Yingruo Li et al.
}

Anonymous Referee \#1

Received and published: 24 July 2019

\section{General comments:}

This manuscript shows the observed concentration of the air pollutions in Beijing before/after the air quality control by the government for the 2014 APEC summit. The manuscript is generally well written, but several points should be clarified. First, although the WTM-observed results are evaluated using other instruments, i.e., moving low percentile method (MLPM), the evaluation of the MLPM itself is unclear. Second, the quantification to evaluate the results is inadequate in some parts like Figures 7 and 8. Third, $\mathrm{O} 3$ is the secondary component, so its discussion should be separated 
from that in the primary components, i.e., NO, (NOx), BC, CO and SO2. In overall, the manuscript would be acceptable for publication if these comments can be satisfactorily addressed.

Specific comments:

P.1, L27-28: I don't think the current manuscript adequately shows the feasibility and stability of the WTM. Also, which point of the WTM is useful for the evaluation of pollution control policies? These are unclear.

P.5, L24-25: What is the moving low percentile method (MLPM)?

P.5, L33-L36: Do the authors discuss an impact of the assumption on main results?

P.6, L17-L19: The authors use "comparable", but this is not suitable for CO and SO2 shown in L20. The difference in $\mathrm{CO}$ and SO2 between this study and the reference is more than twice. Also, I think the level of $\mathrm{O} 3$ in China is relatively lower than that in the other regions.

\section{P.8, L12-L13: How about SO2 and O3?}

P.8, L30-L31: There is no information about the statistic metrics such as bias, correlation coefficient, and root-mean-square-error. Please show them for the comparison. In addition, the uncertainties are estimated in Tables 3 and 4, but actually how are they calculated? I don't exactly understand the meaning of "the SD of on-road concentrations estimated using different schemes in the WTM divided by the mean pollutant concentration" on Tables 2 and 3. What is the different scheme? Does the SD determine the uncertainty?

Table 5: Does it show the ratio of $\mathrm{CH}$ to the sum of $\mathrm{CH}$ and $\mathrm{CL}$ ? If so, this result may show a failure of the decomposition of $\mathrm{CH}$ and $\mathrm{CL}$. I expected the value in full-APEC is lower than that in the other period, but it is not. Perhaps, the total amount of the sum of $\mathrm{CH}$ and $\mathrm{CL}$ in full-APEC is also lower than that in the other period, so that the proportion in full-APEC is not lowest among the periods. In this sense, I don't understand what 
the main message in this table is.

Figure S4: Are the R values corrected? They look high, even though the points are ACPD well scattered. Please check them again. The authors can insert the statistic metrics into Figures 7 and 8.

Technical comments:

P.1, L23-L24 \& P.10, L16-L18: 50\% $\rightarrow$ 50.0\%

P.3, L4: "In Sect. 3" can be removed.

P.3, L31: Where is the 5th Ring in Figure 1?

P.6, L11-13: These references may be not exactly related to the current manuscript. Are these important in this manuscript?

P.13, L25: Please add the name of journal.

Figures 4-8: The blue shaded covers the important color. The shaded is not good.

Figure 7: How about SO2 and $\mathrm{O} 3$ ?

Figure 8: How about $\mathrm{BC}$ ?

Table 6: Does it show the sum of $\mathrm{CH}$ and $\mathrm{CL}$ ?

Table 7: Does it show the $\mathrm{CH}$ ?

Interactive comment on Atmos. Chem. Phys. Discuss., https://doi.org/10.5194/acp-2019-310, 2019. 